\title{
Kalman filter design for atmospheric tip/tilt, tip/tilt anisoplanatism and focus filtering on extremely large telescopes
}

L. Gilles*a, H. F. Raynaud ${ }^{b}$, C. Correia ${ }^{c}$, L. Wang ${ }^{a}$, B. Ellerbroek ${ }^{a}$, C. Boyer ${ }^{a}$ and C. Kulcsár $^{b}$ ${ }^{a}$ Thirty Meter Telescope Observatory Corp., 1200 E. California Blvd, MC 102-8, Pasadena, CA 91125, USA; ${ }^{b}$ Laboratoire Charles Fabry de 1'Institut d'Optique - CNRS - Université Paris-Sud Orsay, RD 128, 91127 Palaiseau CEDEX, France; ${ }^{\mathrm{c} C e n t e r}$ for Astrophysics, University of Porto, Porto, Portugal

\begin{abstract}
This paper discusses Kalman filter design to correct for atmospheric tip/tilt, tip/tilt anisoplanatism and focus disturbances in laser guide star multi-conjugate adaptive optics. Model identification, controller design and computation, command oversampling and disturbance rejection are discussed via time domain analysis and control performance evaluation. End-to-end high-fidelity sky-coverage simulations are presented by Wang and co-authors in a companion paper.
\end{abstract}

Keywords: Kalman filter, Linear Quadratic Gaussian control, vibration, adaptive optics

\section{INTRODUCTION}

Much research has been performed in past several years on Linear Quadratic Gaussian (LQG) control for adaptive optics (AO) systems to reject atmospheric turbulence and vibration lines [1]-[11]. This paper focuses on Kalman filter design to control tip/tilt, tip/tilt anisoplanatism and focus disturbances in laser guide star (LGS) multiconjugate adaptive optics (MCAO), based on previous work described in Ref.[11] and Ref.[8]. The main deviations from earlier work reside in adopting (i) different disturbance shaping filters, (ii) a model identification strategy now based on fitting empirical power spectral densities (PSDs), (iii) a different state vector representation, and (iv) a different state noise covariance matrix identification method. The manuscript is organized as follows: Section 2 describes the LQG control model and the state-space parameters identification method, Section 3 presents simulation results and Section 4 concludes.

\section{KALMAN FILER DESIGN AND IDENTIFICATION}

\subsection{Overview}

Table 1 provides a top-level summary of the off-line steps involved in the computation of the Kalman gain matrix, whereas Table 2 describes the online steps. CT, DT, ID and DARE refer respectively to "continuous time", "discrete time", "identification" and "discrete algebraic Riccati equation". Further details are provided in Section 2.2.

\begin{tabular}{|l|l|}
\hline CT state-model ID & Model formulation \\
\hline & Parameter estimation via empirical PSD fitting \\
\hline & $\begin{array}{l}\text { State-vector covariance matrix computation via CT Lyapunov equation with } \\
\text { state-noise covariance adjusted to yield the desired disturbance covariance matrix }\end{array}$ \\
\hline $\begin{array}{l}\text { DT state-model ID \& extension to } \\
\text { include WFS measurement model }\end{array}$ & Model formulation \\
\hline & State-noise covariance matrix computation via routine matrix algebra \\
\hline & Measurement-noise covariance matrix estimation \\
\hline Kalman gain matrix computation & \\
\hline & DT state-vector estimation error covariance matrix estimation from DARE. \\
\hline & Kalman gain matrix computation from state-vector error covariance matrix \\
\hline
\end{tabular}

Table 1 Tope-level description of the off-line steps involved in the computation of the Kalman gain matrix. 


\begin{tabular}{|l|l|}
\hline State-vector Estimation Stage & \\
\hline & Pseudo open loop measurement computation \\
\hline & Innovation computation \\
\hline & State estimate update \\
\hline Control Stage & State prediction \\
\hline & \\
\hline & State estimate temporal up-sampling via prediction \\
\hline & Temporal averaging \\
\hline
\end{tabular}

Table 2 Tope-level description of the online LQG controller implementation.

\subsection{Turbulence Disturbance Model}

The following $2^{\text {nd }}$-order stochastic ordinary differential equation (ODE) is assumed to describe each turbulence disturbance mode $d_{i}(i=1, \cdots, 6)$ :

$$
\begin{aligned}
& d_{i}=g_{1, i} y_{i}+g_{2, i} \dot{y}_{i}, \\
& \ddot{y}_{i}+2 \xi_{i} \omega_{i} \dot{y}_{i}+\omega_{i}^{2} y_{i}=\omega_{i}^{2} \epsilon_{i}(t),
\end{aligned}
$$

where $\epsilon_{i}$ is white state-noise with variance $\sigma_{\epsilon_{i}}^{2}$. In Laplace space, the associated transfer function ("shaping filter") is given by the following expression:

$$
\frac{d_{i}(s)}{\epsilon_{i}(s)}=H_{d_{i}}(s)=\frac{\omega_{i}^{2}\left(g_{1, i}+g_{2, i} s\right)}{s^{2}+2 \xi_{i} \omega_{i} s+\omega_{i}^{2}} .
$$

In terms of the PSDs, we have:

$$
\operatorname{PSD}_{d_{i}, \text { model }}(v)=\left|H_{d_{i}}(s=2 j \pi v)\right|^{2} \sigma_{\epsilon_{i}}^{2} .
$$

By introducing the following state-vector:

$$
x_{d_{i}}=\left(\begin{array}{c}
y_{i} \\
\dot{y}_{i} / \omega_{i}
\end{array}\right) \text {, }
$$

(2.1) is rewritten in state-space form as follows:

$$
\begin{aligned}
& \dot{x}_{d_{i}}(t)=A_{d_{i}} x_{d_{i}}(t)+B_{d_{i}} \epsilon_{i}(t), \\
& d_{i}(t)=\mathrm{C}_{d_{i}} x_{d_{i}}(t), \\
& A_{d_{i}}=\left(\begin{array}{cc}
0 & \omega_{i} \\
-\omega_{i} & -2 \xi_{i} \omega_{i}
\end{array}\right), B_{d_{i}}=\left(\begin{array}{c}
0 \\
\omega_{i}
\end{array}\right), C_{d_{i}}=\left(\begin{array}{ll}
g_{1, i} & g_{2, i} \omega_{i}
\end{array}\right) .
\end{aligned}
$$

$A_{d_{i}}, B_{d_{i}}, C_{d_{i}}$ are usually referred to respectively as "state matrix", "input matrix", and "output matrix". For each disturbance mode $d_{i}$, the state matrix parameters are estimated via least-squares fitting of the model PSD (2.3) to an empirical (measured or simulated) PSD:

$$
\left(\hat{g}_{1, i}, \hat{g}_{2, i}, \hat{\omega}_{i}, \hat{\xi}_{i}\right)=\arg \min _{\xi_{i} \geq 1 / \sqrt{2}} \int d v\left[\operatorname{PSD}_{\mathrm{d}_{i}, \text { emp }}(v)-\operatorname{PSD}_{\mathrm{d}_{i}, \text { model }}(v)\right]^{2} .
$$

The 6 disturbance models (2.5) are then concatenated into a single block-diagonal state space model with input $\epsilon(t)$, internal state $x_{d}(t)$ and output $d(t)$ : 


$$
\begin{aligned}
& \dot{x}_{d}(t)=A_{d} x_{d}(t)+B_{d} \epsilon(t), \\
& d(t)=C_{d} x_{d}(t),
\end{aligned}
$$

where $A_{d}=\operatorname{Diag}\left(A_{d_{1}}, \cdots, A_{d_{6}}\right), \quad B_{d}=\operatorname{Diag}\left(B_{d_{1}}, \cdots, B_{d_{6}}\right), \quad C_{d}=\operatorname{Diag}\left(C_{d_{1}}, \cdots, C_{d_{6}}\right), \quad$ and $\epsilon(t) \quad$ is the input multivariable white noise with covariance matrix $\Sigma_{\epsilon}=\left\langle\epsilon \epsilon^{T}\right\rangle$. Since this state-space model is stable, both its internal state $x_{d}(t)$ and output $d(t)$ are guaranteed to be stationary multivariable stochastic processes. More precisely, the covariance matrix of the output of this multivariable shaping filter is given by:

$$
\Sigma_{d}=C_{d} \Sigma_{x_{d}} C_{d}^{T},
$$

where $\Sigma_{x_{d}}=\left\langle x_{d} x_{d}^{T}\right\rangle$ is the unique semi-positive definite solution of the CT Lyapunov equation:

$$
A_{d} \Sigma_{x_{d}}+\Sigma_{x_{d}} A_{d}^{T}+B_{d} \Sigma_{\epsilon} B_{d}^{T}=0
$$

where $\Sigma_{\epsilon}$ is adjusted such that $C_{d} \Sigma_{x_{d}} C_{d}^{T}$ matches the desired value of $\Sigma_{d}$. The latter can be deduced from either theoretical priors, measurements or simulations (in our experiments, the value of $\Sigma_{d}$ was computed from trajectories generated through end-to-end simulations). Note that since $\Sigma_{d}$ is in general not block-diagonal, the same holds for $\Sigma_{x_{d}}$ and $\Sigma_{\epsilon}$.

The next step is to discretize the CT disturbance model (2.7) at the low-order natural guide star (NGS) wavefront sensor (WFS) sampling period, $T_{n g s}$, and to augment the DT state vector $x_{d, n} \equiv x_{d}\left(n T_{n g s}\right)$ with an additional variable needed to model the measurement, namely the average value of vector $d$ over successive intervals $\left[(n-1) T_{n g s}, n T_{n g s}[\right.$, denoted $\bar{d}_{n}$ and expressed as follows:

$$
\bar{d}_{n}=\frac{1}{T_{n g s}} \int_{(n-1) T_{n g s}}^{n T_{n g s}} d(t) d t .
$$

The augmented state vector for the DT disturbance plus WFS measurement model is defined as the concatenation of $x_{d, n}$ and $\bar{d}_{n}$ :

$$
x_{n}=\left(\begin{array}{c}
x_{d, n} \\
\bar{d}_{n}
\end{array}\right),
$$

and the augmented DT state space model is expressed as follows:

$$
x_{n+1}=A x_{n}+v_{n} \text {, }
$$

with

$$
A=\left(\begin{array}{cc}
e^{A_{d} T_{n g s}} & 0_{12 \times 6} \\
Q_{d} & 0_{6 \times 6}
\end{array}\right), \quad Q_{d}=C_{d} \frac{1}{T_{n g s}}\left(e^{A_{d} T_{n g s}}-I_{12 \times 12}\right) A_{d}^{-1} .
$$

The input to this stochastic DT model is a DT Gaussian white noise $v_{n}$ with covariance matrix given by:

$$
\Sigma_{v}=\int_{0}^{T_{n g s}} e^{A_{\text {augt }} t}\left(\begin{array}{cc}
\Sigma_{\epsilon} & 0_{6 \times 12} \\
0_{12 \times 6} & 0_{12 \times 12}
\end{array}\right) e^{A_{\text {aug }}^{T} t} d t
$$

where 


$$
A_{\text {aug }}=\left(\begin{array}{cc}
A_{d} & 0_{12 \times 6} \\
\frac{1}{T_{n g s}} C_{d} & 0_{6 \times 6}
\end{array}\right) .
$$

Note that computing $A$ and $\Sigma_{v}$ from the CT model matrices $A_{d}, B_{d}, C_{d}, \Sigma_{\epsilon}$ involves only routine matrix algebra and solving the CT Lyapunov equation (2.9).

It is convenient to introduce at this point matrices $E_{d}$ and $E_{\bar{d}}$ that extract respectively $x_{d, n}$ and $\bar{d}_{n}$ from the augmented state-space vector, i.e.

$$
x_{d, n}=E_{d} x_{n}, \quad \bar{d}_{n}=E_{\bar{d}} x_{n} .
$$

The Kalman filter is run at the NGS WFS frame rate and directly applied to reconstructed pseudo open loop modes (more details are provided below). An important feature of the controller is that the output commands are upsampled to the higher frame rate LGS loop sampling frequency and applied to the wavefront correctors at the high LGS loop rate. This means that during a single LGS frame, $r=T_{n g s} / T_{l g s} \geq 1$ (assumed to be an integer) commands are applied, where $T_{l g s}$ denotes the LGS WFS sampling period. The filter also models the NGS WFS integration latency (1 NGS frame) and 1 LGS frame of computational latency.

The measurement model used in conjunction with the state-space model (2.12) is assumed of the following form:

$$
z_{n}^{O L}=E_{\bar{d}} x_{n}+R \eta_{n}
$$

where $R$ is the noise-weighted least-squares reconstruction matrix mapping closed loop NGS WFS slopes $s_{n}^{C L}$ into closed loop modal disturbance coefficients, and $\eta_{n}$ denotes the NGS WFS measurement noise at NGS frame $n$ with covariance matrix $\Sigma_{\eta}\left(R \eta_{n}\right.$ is thus the propagated noise onto modal coefficients). This pseudo open loop measurement vector is in practice obtained from $s_{n}^{C L}$ as follows:

$$
\begin{aligned}
& z_{n}^{O L}=R s_{n}^{C L}+\bar{c}_{n-1}^{\text {clipped }}, \\
& \bar{c}_{n-1}^{\text {clipped }}=\frac{1}{r} \sum_{j=0}^{r-1} \bar{u}_{n-1+\frac{j}{r}}^{\text {clipped }},
\end{aligned}
$$

where $\bar{c}_{n-1}^{\text {clipped }}$ and $\bar{u}_{n-1+\frac{j}{r}}$ denote the average LQG controller output clipped command over respectively the time interval $\mathrm{T}_{n g s}\left[n-1, n\left[\right.\right.$ and $(n-1) T_{n g s}+T_{\text {lgs }}[j, j+1[$. It is important to feedback clipped commands (clipping is implemented on the output commands whenever their range exceeds the stroke limit of the correctors) to inform the Kalman filter about such events and keep the loop stable [12].

The innovation is defined as the new information contained in $z_{n}^{O L}$ that is not in previous measurements, or equivalently the error made in estimating $z_{n}^{O L}$ from previous measurements, which is expressed as follows:

$$
\tilde{z}_{n}^{O L}=z_{n}^{O L}-E_{\bar{d}} \hat{x}_{n \mid n-1}
$$

where

$$
\hat{x}_{n \mid n-1}=\left(\begin{array}{c}
\hat{x}_{d, n \mid n-1} \\
\hat{\bar{d}}_{n \mid n-1}
\end{array}\right)=\left\langle x_{n} \mid z_{n-1}^{O L}, \cdots, z_{1}^{O L}\right\rangle,
$$

and $\langle x \mid y\rangle$ denotes the expectation of Gaussian variable $x$ conditioned to Gaussian variable $y$. The state estimate update is expressed as follows:

$$
\hat{x}_{n \mid n}=\hat{x}_{n \mid n-1}+K_{\infty} \tilde{z}_{n}^{O L}
$$


where

$$
\begin{aligned}
& \hat{x}_{n \mid n}=\left(\begin{array}{c}
\hat{x}_{d, n \mid n} \\
\hat{\bar{d}}_{n \mid n}
\end{array}\right)=\left\langle x_{n} \mid z_{n}^{O L}, \cdots, z_{1}^{O L}\right\rangle=\hat{x}_{n \mid n-1}+\left\langle x_{n} \mid \tilde{z}_{n}^{O L}\right\rangle, \\
& \left\langle x_{n} \mid \tilde{z}_{n}^{O L}\right\rangle=K_{\infty} \tilde{z}_{n}^{O L} .
\end{aligned}
$$

The last equality in the first line of (2.22) follows from a fundamental property of the conditional mean and innovation and is at the crux of the Kalman filter [13]. $K_{\infty}$ denotes the steady-state Kalman gain matrix, and is expressed as follows:

$$
K_{\infty}=\Sigma_{\infty} E_{\bar{d}}^{T}\left(E_{\bar{d}} \Sigma_{\infty} E_{\bar{d}}^{T}+R \Sigma_{\eta} R^{T}\right)^{-1}
$$

where $\Sigma_{\infty}=\left\langle\left(x_{\infty}-\hat{x}_{\infty}\right)\left(x_{\infty}-\hat{x}_{\infty}\right)^{T}\right\rangle$ denotes the steady-state stave vector estimation error covariance matrix. The measurement noise covariance matrix, $\Sigma_{\eta}$, is computed from an estimate of the NGS WFS signal level during the observation and an estimate of the detector read noise. The error covariance matrix $\Sigma_{\infty}$ is computed off-line as the solution of a filtering (DARE), and is a nonlinear function of $A, E_{\bar{d}}, \Sigma_{v}$ and $R \Sigma_{\eta} R^{T}$. Finally, the state estimate $\hat{x}_{n \mid n}$ is propagated forward in time by the state matrix as follows:

$$
\hat{x}_{n+1 \mid n}=A \hat{x}_{n \mid n} .
$$

Equations (2.18), (2.19), (2.21) and (2.24) constitute the Kalman filter recursion, which is initialized with null vectors, i.e. $\hat{x}_{0 \mid 0}=\hat{x}_{100}=0$, and $\bar{c}_{0}^{\text {clipped }}=\bar{c}_{1}^{\text {clipped }}=0$. The Kalman filter model needs to be updated as conditions change to account for changes in measurement noise and turbulence conditions.

The LQG controller output command is computed during the control stage which follows the above estimation stage. The control stage consists of extracting $\hat{x}_{d, n \mid n}$ from $\hat{x}_{n \mid n}$ and up-sampling the estimate to the LGS loop frame rate. The extraction step is simply expressed as:

$$
\hat{x}_{d, n \mid n}=E_{d} \hat{x}_{n \mid n},
$$

and the up-sampling step is composed of a prediction step:

$$
\hat{x}_{d, n+\frac{j+1}{r}}=e^{A_{d}(j+1) T_{g s}} \hat{x}_{d, n \mid n}, \quad j=0, \cdots, r-1
$$

and an averaging step:

$$
\bar{u}_{n+\frac{j+1}{r}}=C_{d} \frac{1}{T_{l g s}}\left(e^{A_{d} T_{g s}}-I_{12 \times 12}\right) A_{d}^{-1} \hat{x}_{d, n+\frac{j+1}{r} \mid n} .
$$

\section{SIMULATION RESULTS}

We have compared the performance of the Kalman filter model described in Section 2 to an optimized type 1 integral controller for the 4 sample NGS asterisms described in Ref.[14], for the Thirty Meter Telescope (TMT) Narrow Field InfraRed Adaptive Optics System (NFIRAOS) [15], [16]. Sampling periods, signal-to-noise ratio (SNR) and measurement noise equivalent angle (NEA) are listed in Table 3. 


\begin{tabular}{|l|l|c|c|c|c|c|c|}
\hline & \multicolumn{2}{|l|}{$\begin{array}{l}\text { sampling } \\
\text { period/1.25ms }\end{array}$} & \multicolumn{3}{l|}{ SNR @ sampling period } & \multicolumn{3}{l|}{ NEA (mas) @ sampling period } \\
\hline & & TTF & TT1 & TT2 & TTF & TT1 & TT2 \\
\hline ast1 & 8 & 3 & 8 & 8 & 7 & 1 & 1.2 \\
\hline ast2 & 10 & 18 & 6 & 4 & 1.8 & 1.9 & 4.2 \\
\hline ast3 & 9 & 7 & 25 & 3 & 5 & 0.4 & 2.9 \\
\hline ast4 & 12 & 12 & 52 & 4 & 2.9 & 0.3 & 4.2 \\
\hline
\end{tabular}

Table 3 Main NGS WFS parameters.

The Kalman filter or type 1 controllers run at the sampling frequency specified in Table 3, but their commands are upsampled to the LGS loop sampling frequency of $800 \mathrm{~Hz}$. To simulate the integrator using open-loop slopes, we implement the filter corresponding to its equivalent disturbance rejection function, namely:

$$
c_{n}=c_{n-1}+g\left[z_{n}^{O L}-\left(1-\frac{1}{r}\right) c_{n-1}-\frac{1}{r} c_{n-2}\right] .
$$

Command up-sampling for the integrator is implemented using a zero-order hold $(\mathrm{ZOH})$ model. We consider 6 modal disturbances: 5 NGS modes consisting of tip, tilt, and 3 plate scale (PS) (i.e. tilt anisoplanatism) modes, plus focus. The tip/tilt and PS modes are invisible to the LGS wavefront sensors (WFSs), and projected out from the output of the NFIRAOS high-order LGS loop to reduce cross-coupling with the low-order NGS loop, an architecture called "adhoc split tomography" (AHST) [17]. Figure 1 compares open loop with LGS compensated RMS values, averaged over a 17 arcsec x 17 arcsec field of view (FoV). Science-based and NGS gradient-based weightings are considered for the NGS removal step on the NFIRAOS high-order LGS loop. It is seen that closing the LGS loop with science weighting reduces significantly the PS RMS values over the narrow $17 \operatorname{arcsec} \mathrm{x} 17 \operatorname{arcsec}$ FoV. 30,000 time steps long time histories (PSDs down to $0.027 \mathrm{~Hz}$ ) were obtained using MAOS [18] for the 7-layer Mauna Kea median turbulence profile [14] . Each phase screen was sampled at $1 / 64 \mathrm{~m}$ to capture high-spatial frequency errors, and had a $512 \mathrm{~m}$ width (i.e. $32 \mathrm{~K} \times 32 \mathrm{~K}$ phase points per screen). The 7 screens are stored to disk ( $8 \mathrm{~GB} / \mathrm{screen}$ in double precision) and are periodically readout as the simulation progresses. The resulting modal PSDs (simulation and model fit) are displayed in Figure 3 and Figure 3.

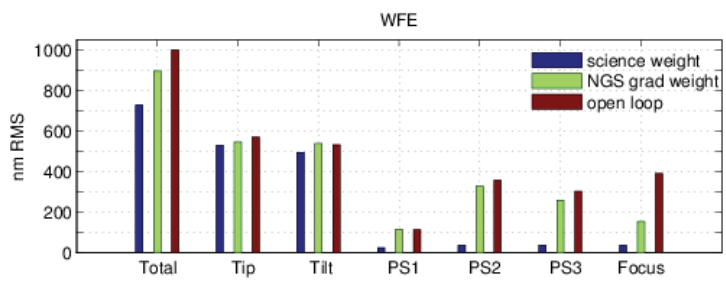

Figure 1: RMS disturbance WFE breakdown.
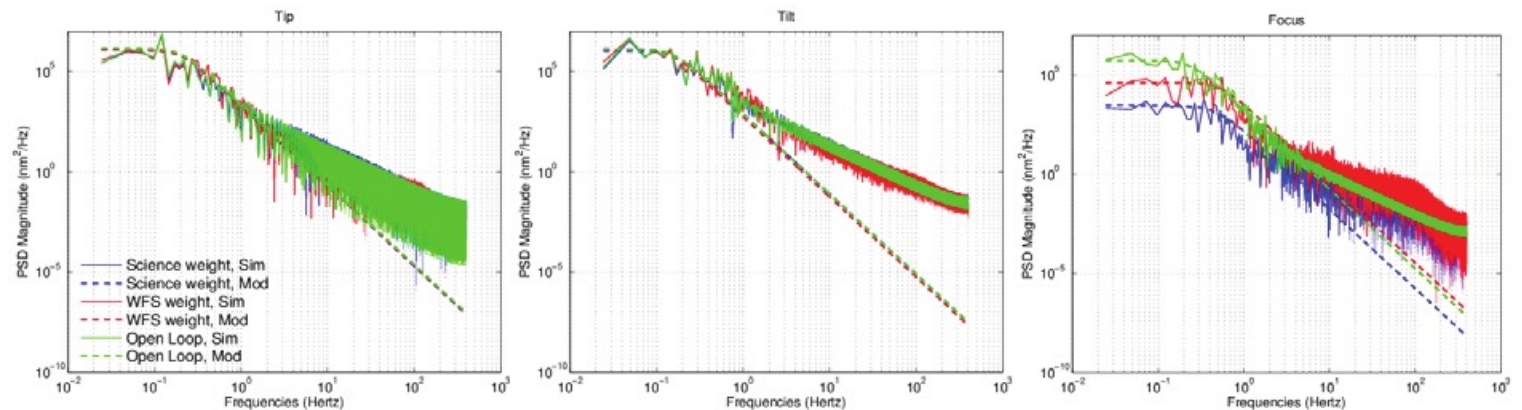

Figure 2: Tip/tilt and focus PSDs: simulation (solid) and model fit (dashed). 

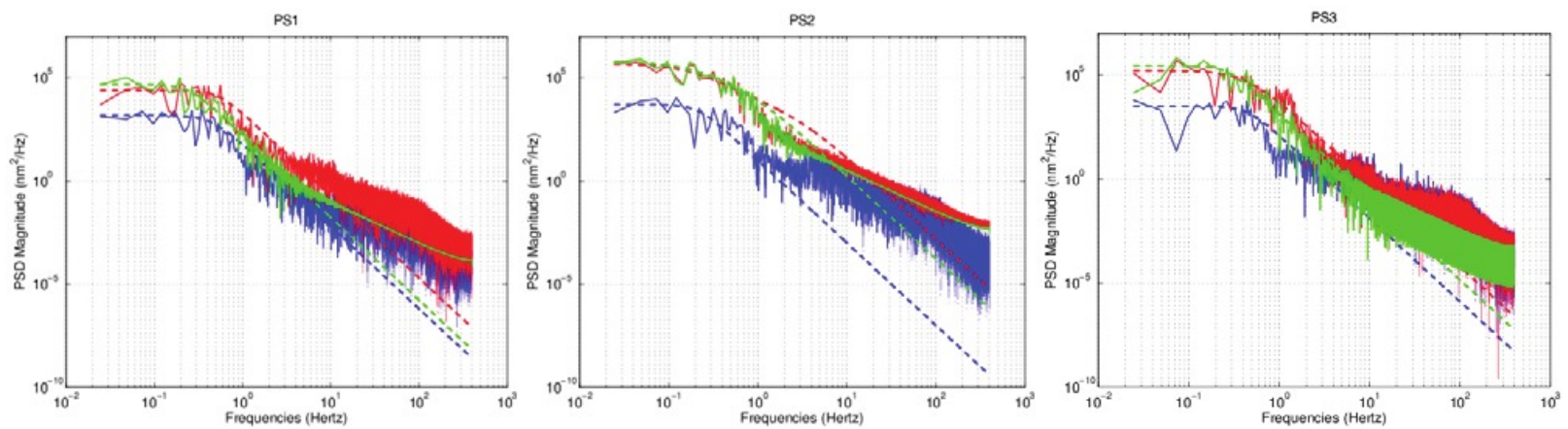

Figure 3: Plate scale PSDs: simulation (solid) and model fit (dashed).
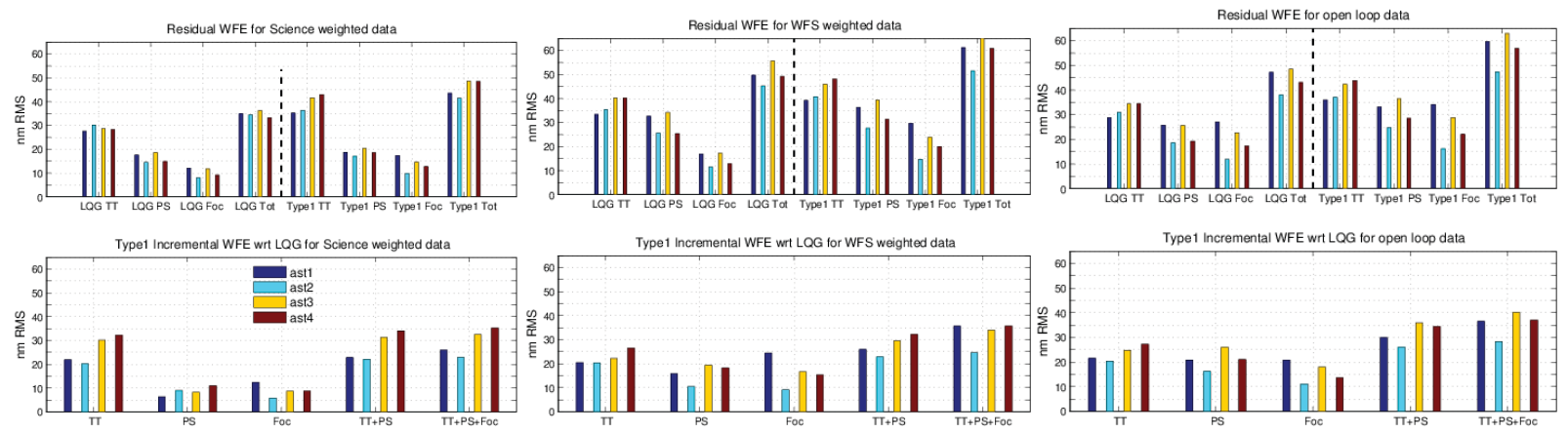

Figure 4: Performance results.

Command performance results are summarized in Figure 4 and Table 4 for the 37.5s long simulations $(30,000$ steps of $1.25 \mathrm{~ms}$ each). It is seen that LQG outperforms optimized type1 control in all cases and modes considered, which is consistent with earlier results [11]. Sample trajectories for the open loop data are displayed in Figure 5 and Figure 6. Note that both type 1 and LQG controllers upsample their output to $800 \mathrm{~Hz}$, and that separate gains were applied to the tip/tilt, PS and focus modes for the former. Finally, sample optimized gains and WFE curves are provided in Figure 7, which shows that the LQG controller is very robust against uncertainties in the RMS state noise value.

\begin{tabular}{|l|l|l|}
\hline & NGS modes & NGS modes + focus \\
\hline Science weight & \multicolumn{2}{|c|}{$20-30$} \\
\hline Gradient weight & $23-33$ & $25-35$ \\
\hline Open loop & $26-36$ & $28-40$ \\
\hline
\end{tabular}

Table 4 Optimized type1 control incremental WFE (nm RMS in quadrature) compared to LQG control.
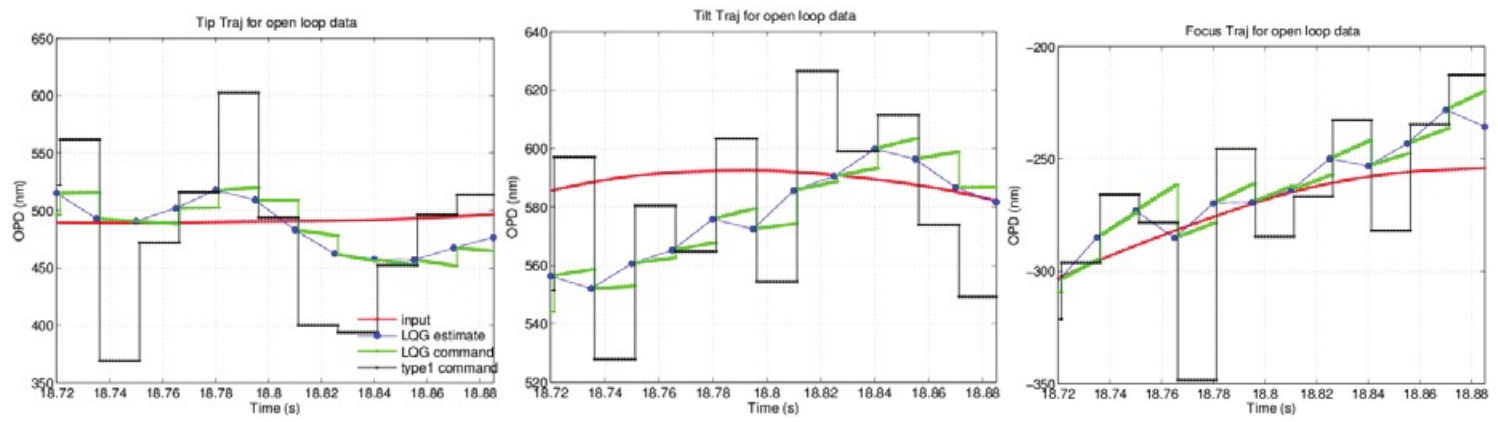

Figure 5: Sample tip/tilt/focus trajectories. 

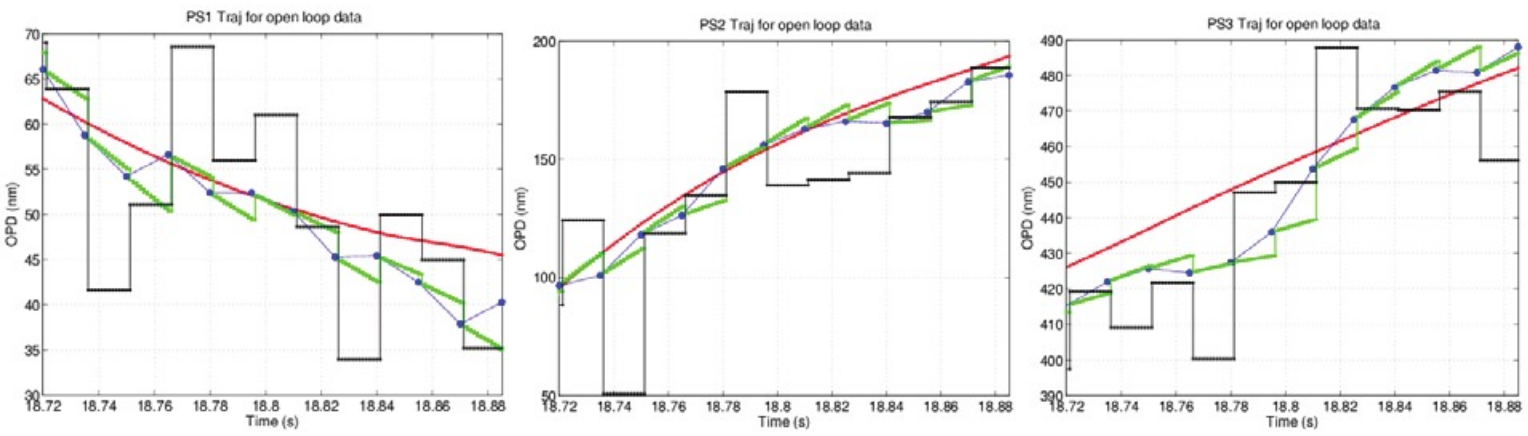

Figure 6: Sample plate scale trajectories.
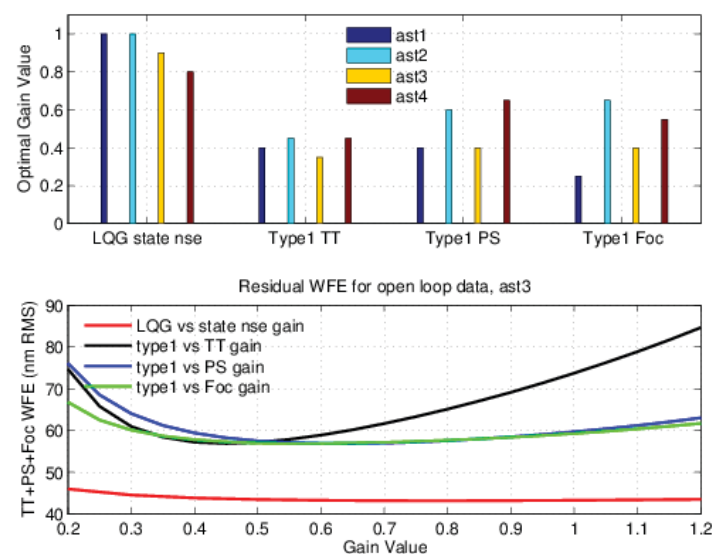

Figure 7: Top: optimal type1 modal gains and LQG state noise gain. Bottom: sample WFE cross-sections versus gains.

\section{SUMMARY}

We have shown that proper Kalman filter design and tuning can lead to significant performance gains compared to standard optimized type 1 integral control to reject stochastic turbulence-induced disturbances. For TMT's first light AO system, NFIRAOS, performance gains up to 40nm RMS are predicted for the tip/tilt, tilt anisoplanatism and focus modes for the AHST control architecture under median turbulence conditions. Detailed end-to-end Monte Carlo sky coverage simulations are presented in a companion paper [19]. Kalman filtering applied to the more optimal minimum variance split tomography (MVST) architecture [20] will be evaluated in a follow up publication.

\section{ACKNOWLEDGMENT}

The authors gratefully acknowledge the support of the TMT collaborating institutions. They are the Association of Canadian Universities for Research in Astronomy (ACURA), the California Institute of Technology, the University of California, the National Astronomical Observatory of Japan, the National Astronomical Observatories of China and their consortium partners, and the Department of Science and Technology of India and their supported institutes. This work was supported as well by the French National Research Agency through project CHAPERSOA ANR-09-BLAN-016201, the European Research Council Marie Curie Intra-European Fellowship FP7-PEOPLE-2011-IEF 300162, the Gordon and Betty Moore Foundation, the Canada Foundation for Innovation, the Ontario Ministry of Research and Innovation, the National Research Council of Canada, the Natural Sciences and Engineering Research Council of Canada, the British Columbia Knowledge Development Fund, the Association of Universities for Research in Astronomy (AURA), the U.S. National Science Foundation and the National Institutes of Natural Sciences of Japan. 


\section{REFERENCES}

[1] Kulcsár, C., Raynaud H.F., Petit C., Conan J.M., de Lesegno P.V., "Optimal control, observers and integrators in adaptive optics," Opt. Exp. 14, 7464-7476 (2006).

[2] Kulcsár, C., Raynaud H.F., Petit C., Conan J.M., "Minimum variance control in presence of actuator saturation in adaptive optics," Proc. SPIE 7015, 70151G (2008)

[3] Kulcsár, C., Raynaud H.F., Petit C., Conan J.M., "Optimal AO control with NGS/LGS wavefront sensors: the multirate case," Proc. SPIE 7736, 773614 (2010).

[4] Conan, J.M., Raynaud H.F., Kulcsár C., Meimon S. and Sivo G., "Are integral controllers adapted to the new ear of ELT adaptive optics?" Proc. AO4ELT2, http://ao4elt2.lesia.obspm.fr (2010).

[5] Meimon S., Petit C., Fusco T. and Kulcsár C., "Tip-tilt disturbance model identification for Kalman-based control scheme: application to XAO and ELT systems," JOSA A27, A122-A132 (2010).

[6] Correia C., Raynaud H.F., Kulcsár C. and Conan J.M., "On the optimal reconstruction and control of adaptive optical systems with mirror dynamics," JOSA A27, 333-349 (2010).

[7] Kulcsár C., Massioni P., Sivo G. and Raynaud H.F., "Vibration mitigation in adaptive optics control," Proc. SPIE $8447,84470 \mathrm{Z}(2012)$.

[8] Correia C., Véran J.P. and Herriot G., "Advanced vibration suppression algorithms in adaptive optics systems," JOSA A29, 185-194 (2012).

[9] Correia C., Jackson K., Véran J.P., Andersen D., Lardière O. and Bradley C., "Static and predictive tomographic reconstruction for wide-field multi-object adaptive optics systems," JOSA A31, 101-113 (2014).

[10] Gilles L., Massioni P., Kulcsár C. and Raynaud H.F., "Distributed Kalman filtering compared to Fourier domain preconditioned conjugate gradient for laser guide star tomography on extremely large telescopes," JOSA A30, 898909 (2013).

[11] Correia C., Veran J.P., Herriot G., Wang L. and Gilles L., "Increased sky coverage with optimal correction of tilt and tilt anisoplanatism modes in laser-guide-star multiconjugate adaptive optics," JOSA A30, 604-615 (2013).

[12] Kulcsár C., Raynaud H.F., Petit C. and Conan J.M., "Minimum variance control in presence of actuator saturation in adaptive optics," Proc. SPIE 7015, 7015G (2008).

[13] Anderson B.D.O. and Moore J.B., “Optimal filtering,” Dover (1995).

[14] Gilles L., Correia C., Véran J.P., Wang L. and Ellerbroek B., "Simulation model based approach for long exposure atmospheric point spread function reconstruction for laser guide star multiconjugate adaptive optics," Appl. Opt.51, 7443-7458 (2012).

[15] Ellerborek B., Adkins S., Andersen D. et al., "TMT adaptive optics program status report,” Proc. SPIE 8447, 84471J (2012).

[16] Boyer C, Adkins S., Andersen D. et al., "Adaptive optics program at TMT," Proc. SPIE, this conference (2014).

[17] Gilles L. and Ellerbroek B., "Split atmospheric tomography using laser and natural guide stars," J. Opt. Soc. Am. A 25, 2427-2435 (2008).

[18] Wang L. and Ellerbroek B., "Computer simulation and real-time control of ELT AO systems using graphical processing units," Proc. SPIE 8447, 844723 (2012).

[19] Wang L, Gilles L, Ellerbroek B. and Correia C., "Physical optics modeling of sky coverage for TMT NFIRAOS with advanced LQG controller," Proc. SPIE, this conference (2014).

[20] Gilles L., Wang L. and Ellerbroek B., "Minimum variance split tomography for laser guide star adaptive optics," Eur. J. Control 17, 327-334 (2011). 